\title{
La música en el entorno social del alumnado de Grado en Educación Primaria
}

\section{The music in the social environment of the students of Degree in Primary} Education

\author{
Francisco César Rosa-Napal *, Isabel Romero Tabeayo ** \\ *Universidade da Coruña, **Conservatorio Profesional de Música de A Coruña
}

\begin{abstract}
Resumen
El conocimiento musical del profesorado en formación se ha configurado a partir de diversas circunstancias que han intervenido en su desarrollo personal e intelectual. El paso por los diferentes niveles educativos ha garantizado un contacto inicial con ese lenguaje, mientras que el grado de acercamiento que pueda haber tenido hacia la música - ya sea auditivamente o mediante la práctica instrumentalen el contexto familiar o social, es un factor determinante en la comprensión de los códigos que estructuran ese lenguaje. Este trabajo pretende mostrar de qué manera estuvo presente la práctica musical no obligatoria en la formación musical del futuro profesorado.

Palabras clave: educación musical, formación musical del profesorado, conocimiento profesional docente.
\end{abstract}

\begin{abstract}
The musical knowledge of teachers in training has been configured from different circumstances which have intervened in their personal and intellectual development. Step through the different educational levels has guaranteed an initial contact with that language, while the degree of approach that may have had toward the music by musical hearing or by instrumental practice- in the family or social context, is a determining factor in the understanding of the codes the structure of that language. This paper aims to show how the non compulsory musical practice was present in the musical formation of the future teachers.

Keywords: musical education, teacher training, teacher professional knowledge.
\end{abstract}

\section{Introducción}

En la asignatura de Didáctica de la Expresión Musical, que forma parte de los estudios de Grado en Educación Primaria (GEP), surgen problemas en la consecución de los propósitos de la materia ocasionados por la deficiente formación que presenta el alumnado inicialmente, sobre todo aquel que no tuvo un contacto con la práctica musical en su trayectoria y que cuenta solamente con la formación recibida en los ámbitos oficiales. Esta realidad, sumada a la delimitación temporal de solo un cuatrimestre destinado a la materia según el plan de estudios actual, constituye una evidencia de la necesidad de encontrar estrategias que garanticen mínimamente la calidad de la formación musical del profesorado.
Con el propósito de comprender el proceso de conformación del acervo cultural del alumnado de Grado en Educación Primaria en tres universidades de la Comunidad Autónoma de Galicia, particularmente en cuanto a la expresión musical, se dedicó una sección en esta investigación a analizar la manera en que esa práctica estuvo presente en el marco de sus actividades sociales. Estos datos podrían justificar algunos de los factores que intervienen en el bajo nivel que perciben los participantes sobre sus conocimientos y sus habilidades musicales. En consecuencia, el objetivo específico de este trabajo consiste en la comunicación de los resultados del análisis de la información obtenida en relación con las principales actividades musicales que fueron desarrolladas fuera del ámbito escolar oficial por el alumnado de GEP.

El apartado correspondiente a los resultados, presenta tres bloques que, a su vez derivan de los ítems contestados por el alumnado participante. El primero está enfocado a la formación instrumental no obligatoria, y se centra en la experiencia individual relacionada con la práctica de instrumentos musicales. El segundo bloque recoge las preferencias musicales pasivas que resultan de sus intereses personales y sociales, mientras que el tercero contiene información sobre la práctica musical colectiva. Este último bloque representa los resultados más significativos al describir el grado de implicación que profesorado en formación ha tenido y tiene con la expresión musical.

Los resultados de la indagación sobre la vinculación del alumnado encuestado con la música - como actividad extraescolar - evidencian que el apoyo que podrían aportar las actividades relacionadas con la música en las etapas preuniversitarias es muy escaso, por lo que es imprescindible reflexionar sobre las causas y las posibles soluciones del problema.

\section{Fundamentación teórica}

\section{Presencia de la música en el contexto social}

La música es uno de los elementos culturales que influyen dinámicamente en la vida social de las personas, en especial en la etapa de la adolescencia. Siguiendo a 
Ruiz (2015), es preciso tener en cuenta dicho planteamiento para valorar su impacto en la formación de la identidad. Esta valoración se refuerza a partir de que la expresión musical, en su dimensión social, presenta dos funciones fundamentales que interactúan entre sí (Ruiz 2015, p. 6):

a. Es elemento socializador, ya que el hecho de que un grupo comparta unos códigos garantiza la relación y la comprensión entre sus miembros.

b. Es elemento diferenciador, ya que el hecho de adoptar unos códigos que no son comunes a toda la sociedad hace que éstos subrayen lo genuino y lo particular de cada comunidad y por ende de cada miembro.

Por su parte, Ramírez (2006, p. 87), afirma que "la música facilita y desarrolla tanto la faceta intelectual como la afectiva", lo que reitera su repercusión en la consolidación de los rasgos culturales e individuales fundamentales de las personas.

Estas consideraciones se refieren al fenómeno musical de manera general, aunque dejando caer su peso sobre la percepción más que sobre la implicación práctica que supone "hacer música". Se deduce de aquí que si la escucha musical participa en la conformación de la identidad individual y social, entonces la posibilidad de crear o interpretar música podría tener la misma, o incluso una mayor incidencia en este sentido. El esfuerzo que supone dominar técnicamente un instrumento, y el compromiso que conlleva pertenecer a agrupaciones musicales de cualquier índole puede potenciar, en gran medida, la influencia de la música en la formación integral.

Considerando que el alumnado participante en este estudio ha transitado por diferentes etapas vitales en las que la música ha tenido un importante papel, podría asumirse que esta manifestación estuvo presente en su desarrollo social y cultural, por lo que sus principales elementos deberían continuar formando parte aquellos conocimientos que conforman esas dimensiones.

\section{La música fuera del ámbito escolar}

La práctica musical no obligatoria tiene diferentes vías de canalización en la actualidad, tanto en el ámbito rural como en el urbano. Dependiendo de los intereses individuales o colectivos es posible realizar actividades musicales interpretativas o creativas.

A partir de un estudio más amplio - del que se deriva este artículo-, en cuanto a las posibilidades de expresión musical fuera del ámbito de la educación obligatoria se obtuvo información sobre la oferta formativa de diferentes instituciones. Aunque solo se trata de centros autorizados u oficiales enfocados a la enseñanza musical, es posible comparar el volumen de las estructuras instructivas disponibles en la región con el resultado de las respuestas del alumnado participante en la investigación, si se tiene en consideración que "la música debe ser entendida como una práctica comunicativa y expresiva fundamental, cercana a cualquier individuo y habitual en cualquier cultura" (Hormigos y Martín, 2004, p. 262).

Según un informe del XVII Encuentro de Consejos Escolares Autonómicos y del Estado (MECD, 2014), en la Comunidad Autónoma de Galicia existen 2 conservatorios superiores de música; 43 conservatorios elementales-profesionales (públicos y privados); 19 centros autorizados y 95 escuelas de música.

Todas estas instituciones promueven la creación de agrupaciones musicales, por lo que debería ser un motivo de alarma que el poco aprovechamiento de esas posibilidades sea tan evidente en el caso de los alumnos y las alumnas de GEP.

\section{Metodología}

De acuerdo a la naturaleza de los objetivos propuestos, la investigación tuvo una orientación cuantitativa, en la que se empleó el cuestionario como instrumento de recogida de datos (Hernández, Fernández, y Baptista, 2006) y que contó con cuatro bloques temáticos:

1. Características sociológicas, culturales y musicales del alumnado de Grado en Educación Primaria.

2. Cultura musical adquirida durante la formación general.

3. La música en la formación inicial del futuro docente generalista

4. Expectativas del alumnado de Grado en Educación Primaria.

Los datos que figurarán en la presentación de los resultados, en el presente trabajo, han sido extraídos del primero de los bloques.

La investigación se llevó a cabo en tres universidades públicas de la Comunidad Autónoma de Galicia y contó, como participantes con el alumnado de Grado en Educación Primaria, una vez concluido su paso por la asignatura correspondiente a Didáctica de la Expresión Musical o aquellas otras denominaciones equiparables.

En la Tabla 1 se presenta la población y la muestra del estudio cuyos participantes contaban con edades entre 19 y 35 años, aunque la mayoría contaba con $20(27,2 \%)$ y con $21(24,5 \%)$ años de edad en el momento del estudio.

Tabla 1

Población y muestra.

\begin{tabular}{lcc}
\hline Universidad & Población & Muestra \\
\hline Universidade da Coruña & 106 & 77 \\
Universidade de Vigo & 155 & 57 \\
$\begin{array}{l}\text { Universidade de Santiago de } \\
\text { Compostela }\end{array}$ & 255 & 168 \\
Total & $\mathbf{5 1 6}$ & $\mathbf{3 0 2}$ \\
\hline
\end{tabular}

\section{Resultados}

\section{Práctica instrumental}

Para poder comprender los resultados de la percepción del alumnado de Grado en Educación Primaria sobre su comprensión de la música, es necesario analizar la manera en que se acercó a esta manifestación durante su vida. La práctica vocal e instrumental es, posiblemente, un buen indicador del acercamiento del alumnado encuestado a la música, ya que en la educación general, en los conservatorios, en las escuelas de música, en 
clases particulares o en las asociaciones culturales es una de las principales actividades de la expresión musical.

En la Tabla 2, los parámetros de la actividad musical práctica están representados por los instrumentos más comunes existentes en la sociedad occidental (Michels, 2004), por lo que ninguno de ellos es totalmente desconocido por el alumnado. Estos instrumentos forman parte de la oferta formativa de instituciones de ámbitos tan diversos como los conservatorios oficiales, las escuelas de música o las asociaciones culturales, y son enumerados como: la voz, considerada aquí como un instrumento corporal tanto individual como colectivo (coro); el piano, que contempla también otros instrumentos que emplean teclados temperados; la percusión, con su amplia gama de posibilidades instrumentales; los instrumentos de cuerda pulsada o frotada; los instrumentos de viento madera o metal, y aquellos otros que, dependiendo de las diferentes regiones que los contemplen como propios (autóctonos), pueden pertenecer a cualquiera de las familias convencionales desde el enfoque organológico.

Tabla 2.

Formación instrumental no obligatoria del alumnado de GEP.

\begin{tabular}{|c|c|c|c|c|c|}
\hline 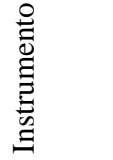 & 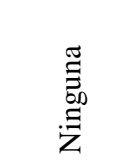 & $\begin{array}{l}\tilde{U} \\
\varnothing \\
\gtrsim \\
\gtrsim \\
ٍ\end{array}$ & 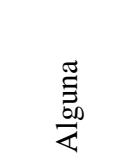 & 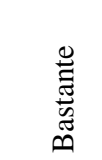 & $\frac{\frac{\pi}{0}}{\stackrel{S}{\Sigma}}$ \\
\hline Voz & $68,80 \%$ & $10,40 \%$ & $9,40 \%$ & $6,70 \%$ & $4,70 \%$ \\
\hline Piano & $78,60 \%$ & $6 \%$ & $5,40 \%$ & $4 \%$ & $6 \%$ \\
\hline Percus. & $71,60 \%$ & $12,20 \%$ & $11,50 \%$ & $2 \%$ & $2,70 \%$ \\
\hline C. puls. & $79,40 \%$ & $8,10 \%$ & $8,10 \%$ & $1,70 \%$ & $2,70 \%$ \\
\hline C.frot. & $93,60 \%$ & $2 \%$ & $2,40 \%$ & $0,70 \%$ & $1,40 \%$ \\
\hline V. mad. & $69,20 \%$ & $8,50 \%$ & $11,20 \%$ & $5,40 \%$ & $5,80 \%$ \\
\hline V. met. & $95,60 \%$ & $2,40 \%$ & $1 \%$ & $0,70 \%$ & $0,30 \%$ \\
\hline Autóc. & $81,60 \%$ & $9,90 \%$ & $3,10 \%$ & $2,40 \%$ & $3,10 \%$ \\
\hline
\end{tabular}

Coincidiendo con los instrumentos propios de la educación musical conocidos como instrumental Orff, los índices más altos del dominio de alguna habilidad instrumental, recae sobre la percusión (de altura determinada o indeterminada), así como los de viento madera (flauta) y la voz. Esta evidencia podría explicar que la práctica instrumental esté relacionada estrechamente con los contenidos curriculares oficiales.

\section{Preferencias musicales}

Las prioridades en el consumo auditivo de la música están marcadas por varios factores. Como se ha expresado anteriormente, y atendiendo a la dualidad diferenciadora y socializadora de la música (Ruiz, 2015), y de todos los elementos extra-musicales asociados a ella, en la Tabla 3 se recoge la frecuencia con que son escuchados algunos géneros o estilos musicales.

Es posible observar que el Pop, seguido del Rock son las manifestaciones más importantes en las preferencias del alumnado participante, mientras que la música coral y la música de cámara son las menos escuchadas. Estas preferencias se originan mayoritariamente fuera del ámbito escolar., mientras otros géneros $\mathrm{o}$ estilos musicales menos preferidos forman parte de los currículos oficiales, aunque no cuentan con una gran presencia en los medios de comunicación. Teniendo en cuenta el impacto de las TIC en la difusión de la música, "los jóvenes experimentan un acercamiento musical dentro de la escuela y fuera de ella, siendo esta última la más constante" (Terrazas, Lorenzo, y González, 2005, p.78).

Tabla 3.

Preferencias musicales.

\begin{tabular}{|c|c|c|c|c|c|}
\hline 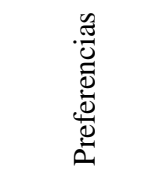 & $\begin{array}{c}\stackrel{\tilde{U}}{\Xi} \\
\text { Z }\end{array}$ & 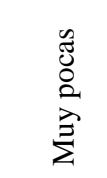 & $\begin{array}{l}\stackrel{\mathscr{Z}}{\Xi} \\
\stackrel{\Xi}{\Xi} \\
\underset{Z}{Z}\end{array}$ & 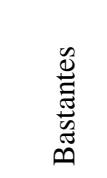 & 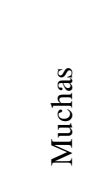 \\
\hline Jazz/Blues & $31,1 \%$ & $26 \%$ & $20,9 \%$ & $13,9 \%$ & $8,1 \%$ \\
\hline Rock & $14,8 \%$ & $15,5 \%$ & $19,2 \%$ & $23,2 \%$ & $27,3 \%$ \\
\hline Pop & $4,7 \%$ & $11,7 \%$ & $17,7 \%$ & $36,8 \%$ & $29,1 \%$ \\
\hline Folk & $29,3 \%$ & $28,6 \%$ & $22,6 \%$ & $13,8 \%$ & $5,7 \%$ \\
\hline Tradic. & $17,8 \%$ & $29,9 \%$ & $28,2 \%$ & $16,8 \%$ & $7,4 \%$ \\
\hline Coral & $51,7 \%$ & $26,8 \%$ & $12,8 \%$ & $6,7 \%$ & $2 \%$ \\
\hline Cámara & $60,4 \%$ & $21,5 \%$ & $9,4 \%$ & $5,7 \%$ & $3 \%$ \\
\hline Sinfónica & $55,7 \%$ & $17,1 \%$ & $15,8 \%$ & $7 \%$ & $4,4 \%$ \\
\hline
\end{tabular}

De acuerdo a estos datos es necesario tener en cuenta, para trabajos posteriores y de más envergadura, que la identificación representativa de los y las participantes, coincide con un tipo de música global (Hernández, 2013) que encierra elementos identificativos comunes a varios géneros y estilos que, de alguna manera, están asociados a la comercialización y a la estandarización de modelos impuestos. Esto explica, en la actualidad, la participación generalizada del público en "eventos musicales que anteriormente se identificaban con determinados grupos bien diferenciados a partir de su identidad cultural y social” (Rosa-Napal, 2015, p. 41).

\section{Práctica musical colectiva}

La pertenencia a alguna agrupación musical, tal y como se ha expresado en la fundamentación teórica, depende del grado de implicación que impulsa al sujeto a comprometerse a desarrollar una actividad en equipo, asumiendo todas sus responsabilidades referentes al cumplimiento de su rol. El compromiso es más patente, si la participación es imprescindible, al tratarse de la ejecución de instrumentos sin los cuales sería imposible: la elaboración de proyectos, los ensayos, las grabaciones o las actuaciones en directo.

En la Tabla 4 se presenta el resultado más revelador de esta exposición, ya que muestra una gran contradicción entre las preferencias musicales pasivas —entendiéndose estas desde la audición-, y las posibilidades reales de actuar creativamente en el ámbito de la expresión 
musical a través de la participación en agrupaciones musicales o en la composición autónoma de nuevas obras en las que se refleje la realidad social en la que están inmersos.

Tabla 4.

Participación en agrupaciones musicales.

\begin{tabular}{|c|c|c|c|c|c|}
\hline 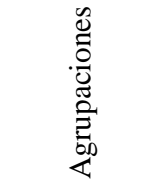 & 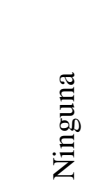 & 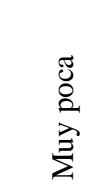 & $\begin{array}{l}\stackrel{\Xi}{E} \\
\text { 茄 } \\
\end{array}$ & 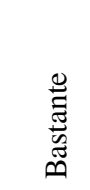 & $\frac{\stackrel{\pi}{0}}{\stackrel{\Sigma}{Z}}$ \\
\hline Jazz/Blues & $94,7 \%$ & $2,7 \%$ & $2,3 \%$ & $0 \%$ & $3 \%$ \\
\hline Rock & $88,6 \%$ & $4 \%$ & $3,7 \%$ & $1 \%$ & $2,7 \%$ \\
\hline Pop & $92,7 \%$ & $2,7 \%$ & $3 \%$ & $0,7 \%$ & $1 \%$ \\
\hline Folk & $90 \%$ & $4,3 \%$ & $2,7 \%$ & $2 \%$ & $1 \%$ \\
\hline Tradic. & $71,7 \%$ & $7,3 \%$ & $11,3 \%$ & $3,3 \%$ & $6,3 \%$ \\
\hline Coro & $70,2 \%$ & $10,4 \%$ & $7,7 \%$ & $4,7 \%$ & $7 \%$ \\
\hline Cámara & $92,7 \%$ & $1,3 \%$ & $2 \%$ & $1,3 \%$ & $2,7 \%$ \\
\hline Sinfónica & $94,3 \%$ & $3 \%$ & $1 \%$ & $0,7 \%$ & $1 \%$ \\
\hline
\end{tabular}

Es relevante que la inquietud activa sobre la música presente estos índices tan bajos. Si se tiene en cuenta que el porcentaje de alumnos y alumnas que no han tenido ningún acercamiento a la expresión musical, en este sentido, es necesario investigar sobre los motivos que propician esta realidad.

Considerando que las preferencias auditivas definidas como mayoritarias (Pop y Rock, Tabla 3) no se reflejen en las prioridades creativas de la mayoría del alumnado participante, es manifiesta una contradicción que evidentemente podría convertirse en un tema independiente de investigación debido a su interés sociológico.

\section{Conclusiones}

Los tres bloques presentados como resultados de la investigación informan, de forma descriptiva, sobre la situación existente en cuanto al acercamiento voluntario a la formación instrumental, las preferencias musicales y la participación creativa que ha tenido el alumnado encuestado en diferentes etapas vitales de forma externa a la educación musical obligatoria.

Como se ha podido observar, esas experiencias no aportan los conocimientos necesarios para consolidar su formación con vistas al empleo de la expresión musical como recurso interdisciplinar en su futura función docente. De haber existido un resultado favorable en cuanto a la formación instrumental extraescolar, este hecho hubiera sido de gran ayuda en la formación didáctico-musical recibida en la titulación de Grado en Educación Primaria, ya que con un mínimo impulso el futuro profesorado contaría con un sólido conocimiento sobre el empleo de la música como recurso educativo.

En otro sentido, la escasa pertenencia a agrupaciones musicales de cualquier índole, se traduce en una dedicación temporal importante durante el curso de la asignatura Didáctica de la Expresión Musical, enfocada solamente a la práctica instrumental colectiva, algo que dificulta la consecución de los verdaderos objetivos de la materia.

Como reflexión final, y considerando que la música, aparte de sus significados culturales, sociales y lúdicos, puede ser frecuentemente empleada como recurso didáctico interdisciplinar, surge la necesidad de recabar más información sobre cuáles son los saberes, las habilidades y las carencias del alumnado de Grado en Educación Primaria en cuanto a ese tipo de expresión, lo que será útil para la concreción de nuevos enfoques formativos en el área y así garantizar su empleo en la futura función docente.

\section{Referencias}

Hernández, D. (2013). Theodor Adorno, elementos para una sociología de la música. Sociológica, $\mathrm{N}^{\circ} 80$, pp. 123-154.

Hernández, R., Fernández, C., Baptista, P. (2006). Metodología de la investigación. Mexico: Mc. Graw Hill.

Hormigos, J. y Martín, A. (2004). La construcción de la identidad juvenil a través de la música. Revista Española de Sociología No 4, pp. 259-270.

MECD (2014). Las enseñanzas artísticas en las comunidades autónomas. XVII Encuentro de Consejos Escolares Autonómicos y del Estado. Disponible en http://www.edu.xunta.es. Recuperado el 23 de agosto de 2015.

Michels, U. (2004). Atlas de música I y II. Madrid. Alianza.

Ramírez, C. (2006). Música, lenguaje y educación: La comunicación humana a través de la música en el proceso educativo. Valencia: Tirant lo Blanch.

Rosa-Napal, F. (2015). La formación musical del futuro profesorado de Educación Primaria. Tesis doctoral. A Coruña: UDC.

Ruiz, A. (2015). El papel de la música en la construcción de una identidad durante la adolescencia. Síneris, $\mathrm{N}^{\mathrm{o}}$ 22, pp. 1-42.

Terrazas, F., Lorenzo, O. y González-Moreno, P. (2005). Consumo y educación musical informal de estudiantes mexicanos a través de TIC. Revista Electrónica de Investigación Educativa, Vol. 17, $\mathrm{N}^{\mathrm{o}}$ 2, pp. 77-88. Recuperado de http://redie.uabc.mx/vol17no2 